\title{
Colour Paper Detection System
}

\author{
A. Pan Thu Tun, B. Khine Myint Mon \\ Department of Electronics, Technological University(Kyaukse), Myanmar \\ Department of Electronics, Technological University(Meiktila), Myanmar
}

\begin{abstract}
This project includes the basic electronic concepts, programming language and compliers, and the use of microcontrollers. The main concepts of this project are the uses of microcontrollers and the programming language. In this project, paper colour detection system detects the different colours and display on LCD. C language is used to assign the function of internal processing of PIC. The source data table in the program had to be displayed the different colours displayed on the LCD screen.
\end{abstract}

Keywords: PIC, LCD, Detection System, LDR, ADC

\section{Introduction}

THIS project is a microcontroller based control system for paper color detection technique. Microcontroller acts as a processing unit and control unit. It obeys the program which is in its program memory. This project is research based project for the future to detect any kind of object which has different colours, size and shape. In this system, $\mathrm{C}$ programming language is used as a heart of command section.

\section{A. Microcontroller}

\section{System Hardware And Components}

Microcontroller are used in the automatically controlled products and devices, such as automobile engine control systems, remote controls, office machines, appliances, power tools and toys. By reducing the size and cost compared to a design that uses a separate microprocessor, memory, and input/output devices, microcontrollers make it economical to digitally control even more devices and processes.

\section{B. Light Dependent Resistor}

Light dependent resistor (LDR) is a light-controlled variable resistor. The resistance of a light dependent resistance decreases with increasing incident light intensity. Light dependent resistor can be applied in light-sensitive detector circuits, and light- and dark-activated switching circuits. A light dependent resistor is made of a high resistance semiconductor. In the dark, a LDR can have a resistance as high as a few mega ohms (M $\Omega$ ), while in the light, a LDR can have a resistance as low as a few hundred ohms.

\section{Liquid-Crystal Display}

A liquid-crystal display (LCD) is a flat panel display, electronic visual display, or video display that uses the light modulating properties of liquid crystals. Liquid crystals do not emit light directly. It is an electronically modulated optical device made up of any number of segments filled with liquid crystals and arrayed in front of a light source (backlight) or reflector to produce images in colour or monochrome. There are many different type of LCD. In this project, J204A is used.

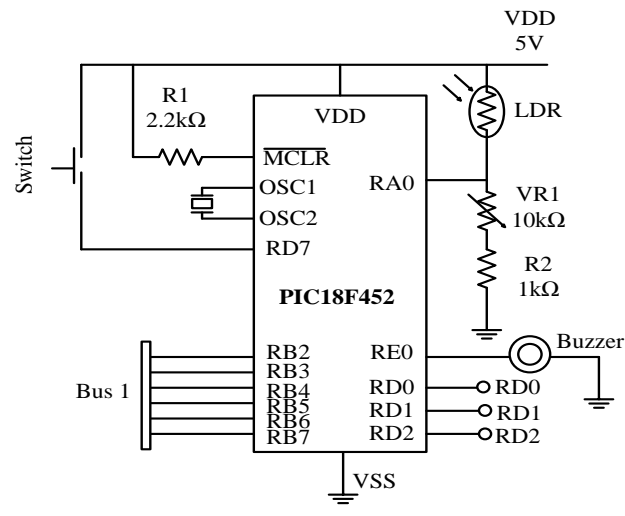

Fig. 1 Control Section of Colour Paper Detection System 


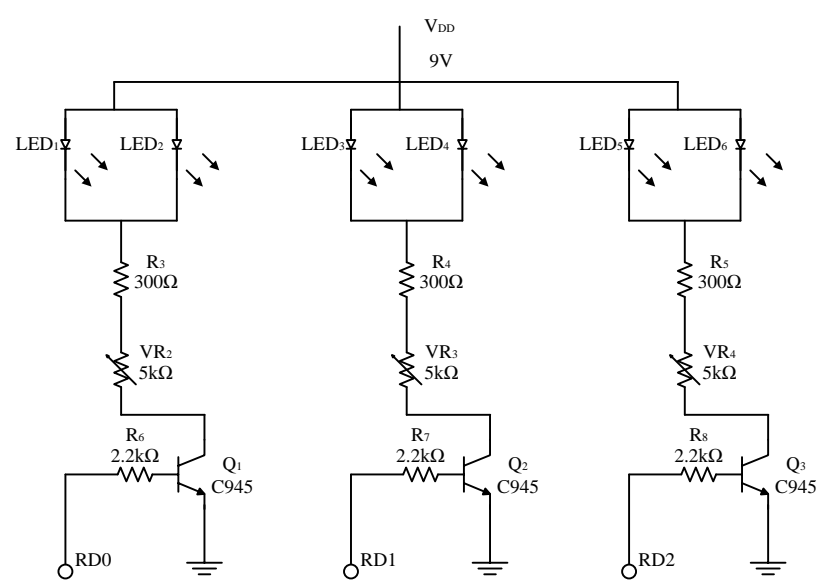

Fig. 2 Input(Sensing) Section of Colour Paper Detection System

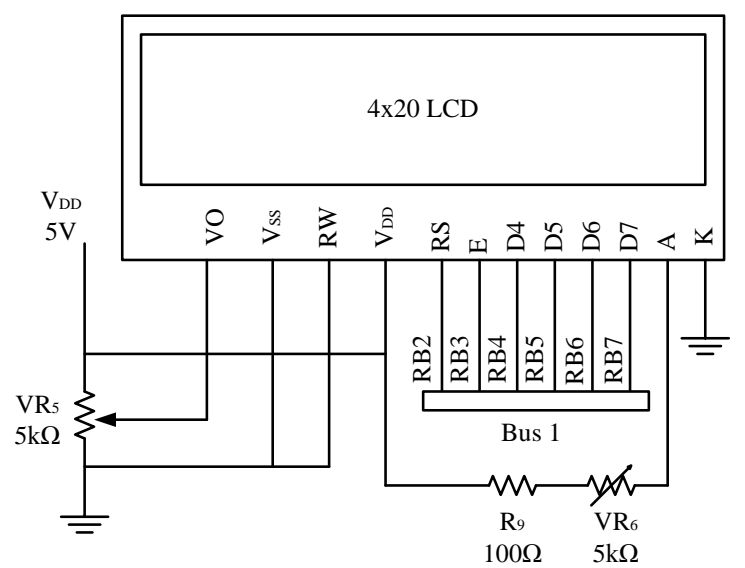

Fig. 3 Display Section of Colour Paper Detection System

\section{Operation Of Input Section}

First, to understand the sensing colours, the colour combination should be known. It is easy to understand how to combine the colours. In theory, the white colour consists of all of basic colours (RGB) with 1:1:1 ratio. This means that the white colour can be got by combining the basic three colours switch each has $100 \%$ brightness. For the black, all of these have $0 \%$ brightness. The red colour has the $100 \%$ of red and $0 \%$ of green and blue. Like the red, the green has $100 \%$ of green and $0 \%$ of red and blue. The blue is also like that.

The yellow has $100 \%$ of red and green and $0 \%$ of blue. The magenta has $100 \%$ of red and blue and $0 \%$ of green. If the combination with $100 \%$ of green and blue with $0 \%$ of red is used, the cyan can be produced. This is the colour combination theory. This is also true for others colours (example. gray, purple, orange, pink and so on).

Second, the colour reflection theory should be obey. The percentages of three basic colours decide that which colour appears. The colour reflection theory is that a coloured particle reflects the colour in terms of three basic colours with percentages of intensity and absorbs some of percentages of basic colours. It reflects a combination of basic three colours and human's eye can detect this as a single colour. For example, the yellow particle absorbs blue approximately $100 \%$ and reflects red and green approximately 100\%. This is most fundamental theory for colour detection.

The sensing the colours is based on this theory. To sense a colour, the different colours are combined with the different ratios of basic colours (RGB). The method for colour sensing is the detecting to know how much each percentage of red, green and blue is combined in a reflected colour. Let see the processing of the system in detail. The different colours have different wavelengths.

In the sensor head, there are six LEDs and these are a pair of red LEDs, green LEDs and Blues. The red LEDs are for red light to detect the red colour percentage. Greens are for the percentage of green and blues are for that of blue. The main sensor component is Light Dependent Resistor (LDR) and it is known that its resistance is inversely proportional to the light intensity which hits against its surface. In fact, the resistance of LDR depends on both density and wavelength of incident light. Different wavelengths cause different changes in resistance of LDR. 
In sensing process, firstly the LDR senses whether a paper is closed into the sensing range or not. If this stage is true, the microcontroller starts the detection stage. It sends the base bias to the transistor Q1 and transistor is turned on the pair of red LEDs for red light. The light from LEDs hits the surface of the coloured paper.

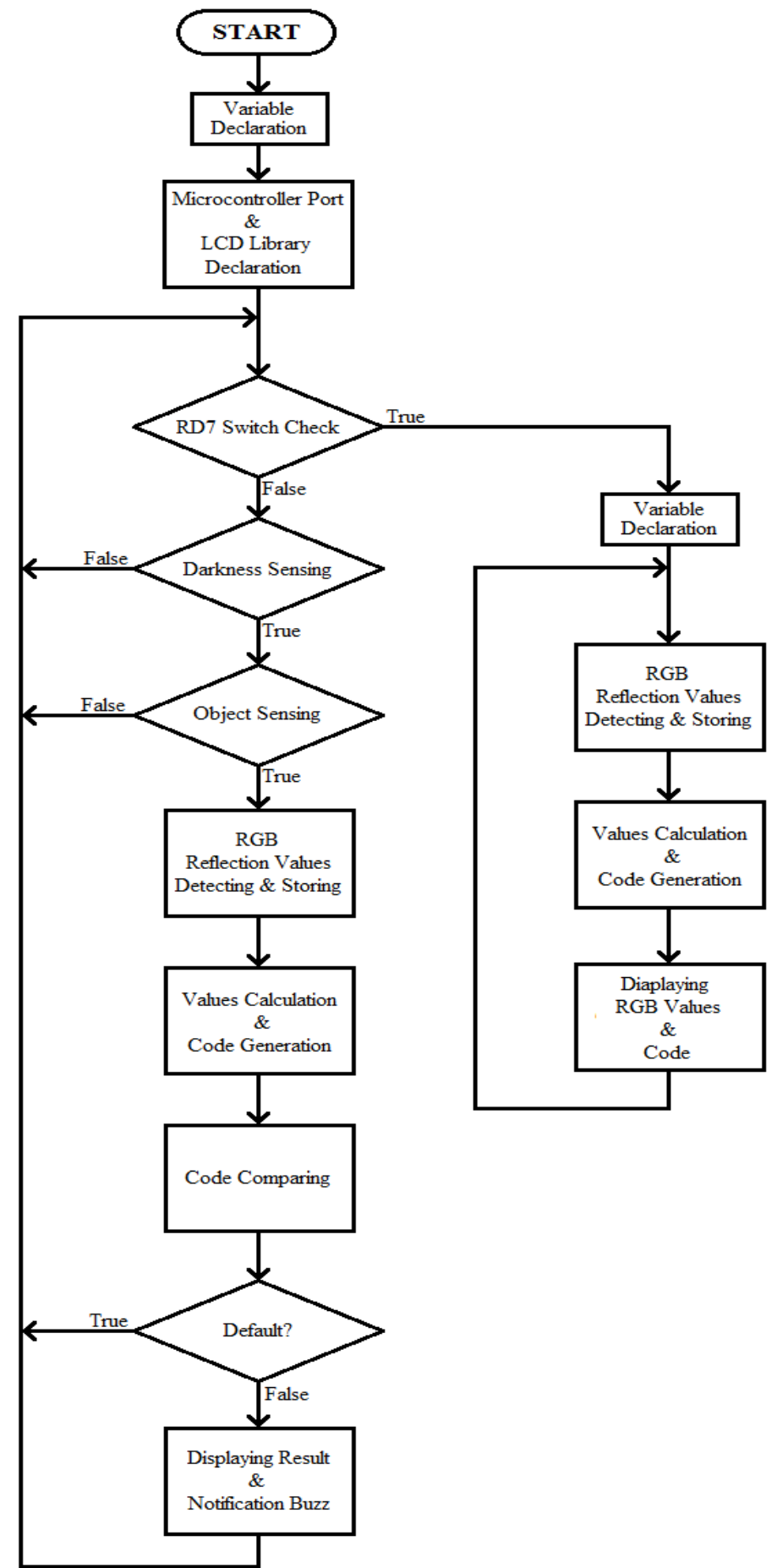

Fig. 4 Flowchart of Software

The paper reflects the red light according to the colour reflection theory and the LDR receives the red light of reflection. Thus, LDR responses that light by changing it resistance. This causes the variation in voltage at pin number 0 of Port A. The analog-to-digital converter (ADC) in microcontroller accepts it from RA0 pin and converts into digital value and saves value into a variable. This is for red response. The next steps are similar to above step for green and blue. 


\section{Operation Of Control Section}

PIC microcontroller obeys the instructions of program written in it. When power to the microcontroller, it starts to execute the program from start point. Then, the variable names are declared and the program is introduced to the microcontroller and the LCD.
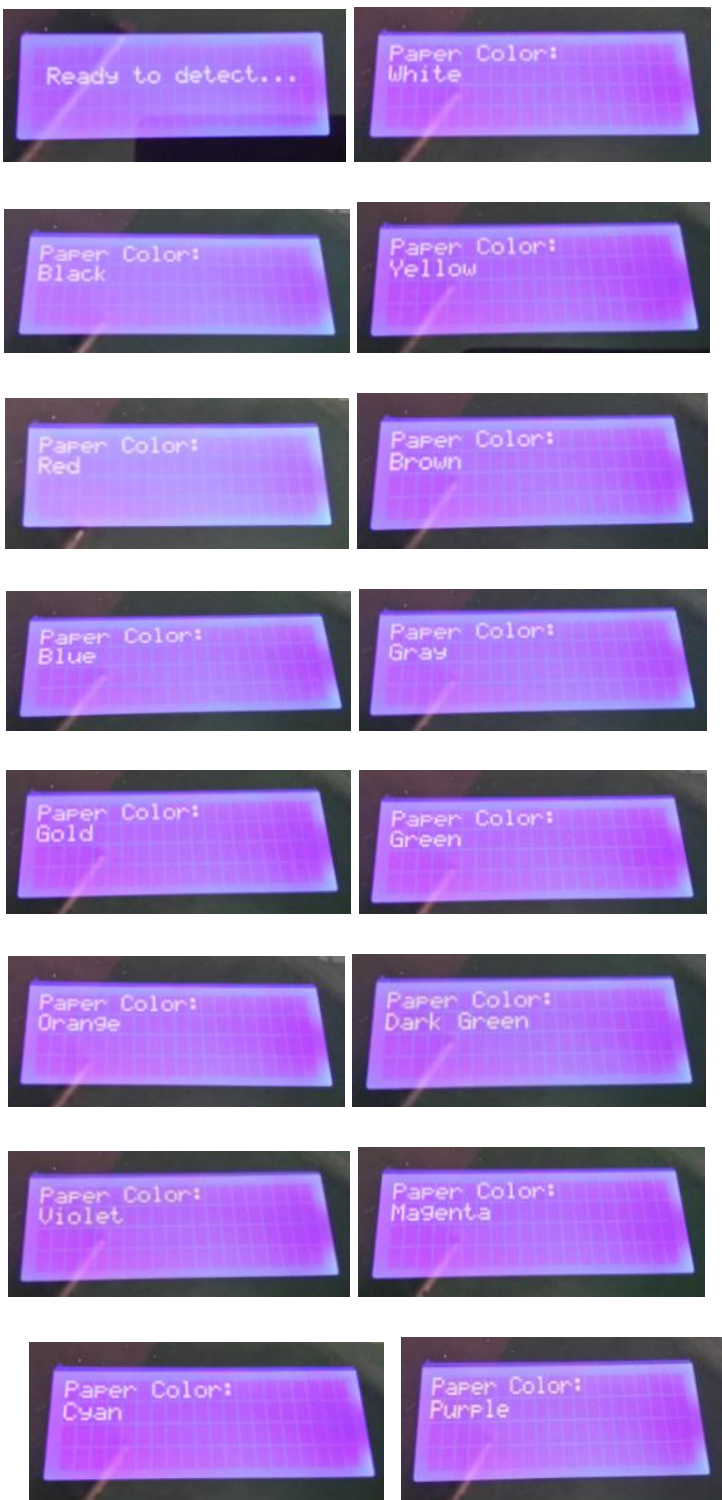

Fig. 5 The output of Colour Paper Detection System

First, the program checks "Test Code Switch" which is fixed in the RD7 of the microcontroller. It is assumed to operate the detection function. Therefore, the switch is at logic state ' 1 ' and the program considers "False" condition. Thus, the next step, darkness sensing, will be processed. If this stage is true, the program will continue the object sensing stage. If an object is sure to detect, the program will starts to detect the colour.

In this step, the reflection value of RGB is stored in a variable array for the next step. Then, in this step, the program calculates and generates a string of code. This result is compared with the colour table in next step. If the colour table has the result code, the colour name will appear on the screen and the user will hear buzz. Otherwise, the program will go back to beginning of the detection stage.

For the "Test Code Function", the code indicator program is executed when RD7 Switch Checking is in true state. In code indicator program, there are no Darkness Sensing and Object Sensing. It calculates the values of RGB and generates codes every second. In this function, the LCD shows the values of RGB and code instead of the name of colour. 


\section{Conclusion}

There are many types of PIC microcontroller. In this project, PIC 18F452 microcontroller, which has 40pins is used because it has built-in A/D Converter. Other types of microcontroller have a smaller size such as PIC 16F84A which contains 18pins but it doesn't contain built-in A/D Converter. So, this will need external A/D converter IC if PIC 18F452 is not used.

\section{Acknowledgment}

Firstly the author would like to thank his parents: (U Kyaw Yin) and (Daw Mya Sein) for their best wishes to join the Engineering University. Special thanks are due to his friend, Khine Myint Mon. The author would like to express his thanks to his all teaching staffs of Department of Electronics, TU(Kse), Myanmar. The author greatly expresses his thanks to all persons whom will concern to support in preparing this paper.

\section{References}

[1]. Predko, M. 2001. Programming and Customizing PIC Microcontrollers, $2^{\text {nd }}$ ed. Printed by Prentice-Hall, Inc.

[2]. Penfold, R. A. 1997. An Introduction to PIC Microcontrollers. $1^{\text {st }}$ ed. Printed by BERNARD BABANI (publishing) LDT.

[3]. Bolyestand, R. Electronic Devices and Circuit Theory. $5^{\text {th }}$ ed. Printed by Prentice-Hall, Inc.

[4]. Iovine, J. 2002. PIC Microcontroller Project Book. Printed by Mc Graw-Hill.

[5]. Win Htet Win,PIC Microcontroller projects in C .

[6]. Microchip. No Date. Data Sheet of PIC18F452 40-Pin 8-bit CMOS FLASH microcontroller.

[7]. <http://www.microchip.com>.

[8]. Nigel Gardner. An Introduction to Programming the microchip PIC in CCS.

Pan Thu Tun was born in 1982, April 1. Graduated in August, 2004 with B.E (Electronics) and finished Master degree on March, 2006 with M.E (Electronics). And then, he is a PhD Holder, Department of Electronics, TU(Kse), Myanmar. He served as a Demonstrator at Mandalay GTC from January, 2001 to January, 2004 when he was attending the Special Engineering Course in MTU. From 4, November, 2004 to 31, March, 2008, he promoted as an Assistant Lecturer of Mandalay Technological University and from 1, April, 2008 to 19, January, 2011, he promoted as a lecturer of Technological University (Pinlon).From 20, January to now, he promoted as Associate Professor. 